\title{
Scientific Methods in Social and Humanitarian Researches
}

\author{
Viktor Viktorovich Skorobogatov*, Inna Vadimovna Yurchenko, Natalya Nikolaevna Yurchenko, \\ Tatyana Yevgenyevna Telyatnik and Oksana Andreyevna Yermolenko
}

\author{
Kuban State University, Russia; monblan@yandex.ru;
}

\begin{abstract}
The article analyzes the importance of methods of scientific disciplines for the research methodology of social and humanitarian processes, and discusses interdependence of life and culture sciences in terms of the complexity theory. It emphasizes that cognition in a social human sphere is based on polyparadigmatic principles which provide both the necessary completeness of the cognitive process and cross-case analysis of the subject under inquiry taking into account specific characteristics of different explanatory models. Quantitative estimations and integral values in scientific methods of social and humanitarian studies provide an opportunity of modeling and predicting social processes and tendencies of political development. The article particularly focuses on the scientific tools for studying micro- and macro-politics in the framework of psychological theories of communication and forms of interaction among influential politicians in the complex multilevel political processes. The author comes to conclusion that an analytical set of research mechanisms based on the principles of naturalistic philosophy can be a basis for system analysis and models of interaction among the main antagonists, taking into account their basic resources and behavior tactics, which in its turn, lets us identify the most likely scenario of development of a situation in a certain time period and on a certain territory. System analysis of structural elements of the social community under inquiry or a certain event line will allow us identifying sources of disfunctionality and latent development of low-intensity conflicts which can involve escalation of confrontation among political actors at the micro and macro levels, which can break down stability, integrity and dynamic equilibrium of the system. The article considers contemporary importance of transformative technologies and engineering capabilities of social theories as a functional problem of the scientific discourse.
\end{abstract}

Keywords: Complexity Theory, Culture Sciences, Integral Values, Life Sciences, Prediction of Social Processes, Quantitative Estimations, Scientific Methods, Social and Humanitarian Studies, Social Modeling

\section{Introduction}

The relevance of the research problem can be explained by the need to empower social and humanitarian cognition by providing epistemological and methodological support for the research process with the help of conceptual approaches of the natural sciences. The contemporary social cognition has three main traditions studying social processes. They are naturalistic, interpretive, and evaluative. In the XX century social and humanitarian knowledge was getting more functions of the actual transformative technologies, and many authors speculated about the problems of engineering capabilities of social theories. For example, sociology is regarded as a science due to the criteria of the level of data objectivity and maximum use of statistical arguments, as well as independence of a researcher from any social and ideological mindsets. At the same time, many authors are still convinced that social and humanitarian cognition, on the one hand, and scientific cognition, on the other, are essentially different ${ }^{1}$. Research methodology of human social behavior has been almost always facing problems of descriptiveness, on the one hand, and excessive abstracting and use of abstract terms, on the other, which, in its turn, has been bringing methodologists back to the primary question: can social and humanitarian sciences follow the principles of natural sciences in conducting researches? $\mathrm{W}$. Dilthey, W. Windelband, and H. Rickert gave a conclusive

\footnotetext{
* Author for correspondence
} 
answer to the question about opposition between nature and culture sciences in their works. Particularly, they argue that natural sciences are nomothetic, and they study common and consistently repeating natural processes, while social sciences are idiographic sciences which study the occasional and the unique. Even though the academia is divided into two camps (some researchers argue that there are no fundamental differences between the sciences, as all of them strive to find the objective truth, while other authors suggest that "single events should be considered as the subject of sociology... and, contrary to natural sciences, search for and formulation of inclusive laws are not of its concern"2), it is suggested that when studying the common and the occasional we need to use both method of social sciences, i.e. understanding, and methods of natural sciences aimed at explanation. In this regard, I shall make my own hypothesis as follows: not denying the logic of those who distinguish natural and social scientific methods, it is necessary to highlight capabilities of the principle of consistency of ultimate distinction and to use it in the methodology of social and humanitarian cognition in order to extend the boundaries of scientific research tools in the process of studying common and occasional social phenomena.

\section{Literature Review}

Literature analysis demonstrates that the analysts in the area of public policy and administration show certain interest in the issue: G. V. Atamanchuk, A. N. Ovcharenko, V. L. Romanov, O. F. Shabrov analyzed social problems following parameters of the A.A. Bogdanov's research "Tectology Universal organizational science" ${ }^{3-7}$.

Analysis of the problem of the use of scientific methods in social and humanitarian cognition regarding correlation between social methods and the methods used in natural sciences ${ }^{8,2}$ was commonly identified with positivism, as well as with the statistical theory of average numbers. Outhwaite W., Boronoev A. O., Deviatko I., Neklessa A. I., Shipunova T. V., and others write about necessity of a new methodology for social sciences. Vasilkova V. V., ${ }^{9}$ and Prigozhin I. and Nikolisa G. ${ }^{10}$ draw our attention to the capabilities of synergetics. Contemporary synergetics studies super complex heterogeneous systems and is based on such world interpretation which assumes its nonlinearity and nonequilibrium. The works which use this explanatory model show that a similar behavior of elements appears when moving from randomness to a certain order - it is a so called cooperative or synergetic effect. As Vasilkova V. V. correctly points out, "a new world image results from a synergetic description... not having become, but becoming, appearing and changing all the time, it evolves according to nonlinear laws..."9. It is necessary to look for a subtle transition from formant state to movement. Classical worldview takes world's rationality as a point of departure, and the science faces a very difficult task, which is to rationally explain an irrational world, to create a rational model of the world.

Problems and factors of achievement of social concertation and progress are analyzed by P. Berger, P. Bourdieu, T. Luckmann, K. Popper, G. Ritzer, and others ${ }^{11-13}$ within different paradigms and on the basis of interdisciplinary principles of social analysis. E. N. Ozhiganov, G. G. Matishov, O. F. Shabrov analyze problems of social modeling and of the design of political situations $s^{6,14,15}$. Thus, a demand for studying problems of use of the scientific methods in social and humanitarian researches has been repeatedly raised in academia, and it needs a more specific study and elaboration.

\section{Method}

\subsection{The Need for a New Methodology of Cognition}

Topicality of studying the changed global social landscape requires a new methodology of cognition and action. As A. I. Nekless a points out, nowadays we need to "change the outdated, which doesn't correspond to the epoch and fundamentals of its analysis language"16, which is generally related to continuous confirmation of the same theoretical statements with additional statistical data and examples. What is fundamentally important for the development of scientific cognition, it is a light of novelty in paradigm parameters of the study of processes and events of the social life.

Division of scientific cognition into two main directions - natural sciences and culture sciences - led to a distinction between the use of research methods of natural processes and social and humanitarian processes. Special characteristic of social and humanitarian knowledge is that the subject and the object of cognition "coincide", which, in its turn, affected the possibility of getting closer to the objective truth, which does not depend on biases caused by social affiliation and other characteristics of the subject of social and humanitarian cognition. We have known about this special characteristic for a long time, and 
there is nothing new in this observation. But the present circumstances require to activate searching for and using of such "analysis language" in social and humanitarian sphere, which could provide a maximum approach of the culture sciences to the nature sciences, first of all, by extending scientific methods and tools. As researchers in the sphere of methodology of social cognition rightly argue, "the challenge to the social sciences came from outside. It started when a movement, which is now called the complexity theory, was born within the science and mathematics"18. A desire to dominate and to have an impact is the main and basic characteristic of the social sphere, and it is the main background characteristic of appearance of new technologies of management of biotic interactions threatening to destroy the relative system equilibrium, which in reality is manifested in the form of state system degradation, violent and illegitimate political regime change including armed conflicts.

Social situation in the process of transition from one system to another is related to both spontaneously appearing and implicit elements of the previously existing system, which cannot disappear instantly and completely. On the one hand, it is distinguished by the prevalence of uncontrolled processes in economy, culture and politics, and on the other hand, by the fact that specific actors who maintain or get power during political and economic transformations and reforms encroaching the right to know and use their dominant position. The potential for confliction and integration is being accumulated in this flow of uncontrolled social processes. And analyzing social changes, we can trace protest fluctuations and support for developing politics expressed by different social and ethnic groups. Action patterns of the subject of these changes, motives and intentions of interest groups, public opinion leaders and decision makers are especially significant in terms of scientific analysis. Conflict confrontation of actors of ethnopolitical relations deepens and weakens simultaneously, thereby confirming the idea of social construction of reality by interior and exterior actors who cooperate and oppose each other.

\subsection{Method of the Social Pressure Theory}

In terms of the issues under inquiry I shall pay attention to the social pressure theory which assumes that when an individual, a social group or a state are under fire and pressure, which is imposed by interested actors with certain resources, this pressure in a relatively confined place leads to "a swabbing effect", to accumulation of excessive energy, which could cause a destructive explosion, if the energy has no way out. Those actors, who are interested in changing political regimes in opponent countries, use suggestive technologies of indirect infusion of the ideas of the so called "self-liberation" in order to weaken the political regimes and bend them to their interests. In such a case, social pressure is defined as a large amount of different changes in psychological state and subjective feelings, motives and emotions, knowledge and beliefs, values and behavioral reactions, which an individual or a society face as a result of real, assumed, or imagined presence of other individuals or their actions ${ }^{18}$. This approach has both micro- and macro-political perspective, if we don't deal with individuals, but with collective communities, including political ones.

Religious, ethnic, ideological heterogeneity of the social community under inquiry leads to contradictory and ambiguous evaluations of political processes, actual events, occurring social phenomena, which causes a so called conflict of interpretations. The role of intellectual groups is especially notorious in understanding the ideological, worldview nature of the conflicts, which are propelled by external political and economic actors, who are somehow interested in a given region, a given territory, in some organization or a social institute. The major characteristic of predictive analytical activities of a researcher is related to eliciting threats, challenges, and risks for a certain system and to defining defense mechanisms in order to preserve and develop the system (taking into account that system preservation is impossible without its continuous development). Thus, social security policy is based on diagnostics and analysis of the current level of social and economic development, prediction of possible crises, and elaboration of preventive social and humanitarian technologies. In relation thereto, activities and recommendations for achieving and increasing the level of social, economic, and political security are elaborated, and their effectiveness is evaluated with the help of a continuous monitoring of the level of security threats and risks, and with the help of respective corrections of the current course with due account for changing external and internal conditions of the carriedout policy. 


\subsection{Research Methods of Social Security Mechanisms: Statistical and Threshold Amount Indicators}

A social security mechanism at the regional level is implemented through practical actions, which include determination of a set of regional interests (provision and maintenance of decent quality of life of the regional population, rational use of the current economic potential, harmony with and integration to the state financial system, as well as the need to protect the interests from different internal and external threats, keeping up the balance with national interests).

The study of social security mechanisms in terms of the current state system problems in some countries demonstrates that social construction per se holds threats to the social structure, established system balance, because "permanent reforms" are as much dangerous as a "permanent revolution". It is possible to decrease conflictogenity of administrative influence with the help of multi-factor analysis of the state policy ${ }^{19}$. A term "war" is more and more used regarding the contemporary information, economic, and technological confrontations, which confirms critical hardness of such conflicts. There are three types of information wars: cognitive (psychological), cyberwars, and behavioral wars ${ }^{20}$. As some authors assume ${ }^{21}$, cognitive and cyberwars are distinguished by target objects and control tools, they aim at changing collective consciousness, entering the opponent's computers and networks in order to inflict him a significant harm. All these types of information wars are led in the electronic networkspace, they have special tools, tactics, and strategy together with the technologies of behavioral wars. Capability of scientific methods in social and humanitarian researches, in particular, can be explained by the internal logic of scientific tools used in the form of analogies, metaphors, and statistical indicators and threshold amount indicators of the system entry into the turbulence state. Thus, for example, the concept dedicated to the use of a spill-over effect in asymmetric and hybrid conflicts is of great importance ${ }^{21}$.

Functional analysis of the structural elements of the social community under inquiry or of a certain event line will allow us to identify sources of disfunctionality and latent development of low-intensity conflicts which can involve escalation of confrontation among political actors at the micro- and macro-levels, which can break down stability, integrity and dynamic equilibrium of the system.

\subsection{Analysis of Micro-Political Mechanisms by Analogy with a Crystal Lattice of Chemical Elements}

We need to reduce scientific thermodynamic methods which describe an irreversible transition of large sets of elements from the less probable to the more probable state, to the system of scientific tools of cognition of the complex multilevel political processes in order to understand the internal mechanisms of micro-politics which tends to confront macro-politics in the framework of psychological theories of communication and forms of interaction among influential politicians.

If we want to consider a special mental characteristic of different ethnic groups, we need to speak about more complex correlation structures, which characterize the state of social feeling of the community under inquiry, its dynamics, and parameters of definition. For example, if the internal ethno psychological intensity of mentality characterizes the common attitude of the emotional and ethnic disposition in conditions of political tensions, then the state of social feeling, moral and spiritual attitude of the community correlates with the political rhetoric and with the picture of the communication network as well. A sustained tension, which occurs in case of a social danger during extremely hard situations of long-running conflicts, information wars, and exposure of psychology of people of the "target state" to one or another stimulus, intensifies unmediated feelings of the people, which can find its manifestations in expressed intensive negative attitude towards the others and in increased aggressive feelings.

This, in its turn, leads to anomy, which equals chaos in the social sphere. But chaos does not disappear if new components of the social order appear: a certain order appears at the macro-level, and disorder, at the micro-level. The basic principle of such approach is to admit relativity of the order and naturally occurred organizational spontaneity, because any system activates its internal protective functions striving for selfpreservation. It does not mean that executives can be inactive. On the contrary, understanding of the nonlinear, multi-probable, complex character of the ordering process lets the elite not to panic about the "system failure" and to search for constructive mechanisms of formation of effective managerial technologies. We can measure the impact on the recipient, the distance weakening degree (both physical and psychological), and the level of 
confidence among individuals and social groups, and the level of impact of surrounding people on the opinion of a certain person according to the dynamic social impact theory ${ }^{22}$.

Formation of social interests is influenced not only by the local events, but by a complex combination of internal and external factors, by macro- and micro-politics, by discourse confrontation of different political segments. From this point of view, effective security policy is an effective management of conflict interaction in the space of ethnic, religious, territorial, social and cultural diversity.

It is possible to find out and afterwards to construct a model of social network communication with different orientation by analogy with crystal lattices of chemical elements.

\subsection{The Problem of Dynamic Social Modeling: the Complex System Model of a Conflict Situation}

The life requires that we pay more careful attention to the cultural and subcultural diversity, special characteristics of political behavior of ethnocultural communities, diagnostics of the current level of political and social security, discovery, prediction and systematization of possible threats to the comprehensive and dynamically developing multi-cultural community.

The complex system model of a conflict situation is based on a certain classification, i.e. in distinguishing those factors, which, according to the analyst, have decisive influence, from a multiple set of events, which have an impact on the conflict. The following components are usually included into the structure of the social dynamic model under inquiry: system factors (structure and functions of the social institutes); subject factors (influential political actors); resource factors (physical, financial, intellectual resources, information technologies, possession of capability to replicate own political projects), and others.

Agent-based modeling of dynamic social systems is of interest, it lets us take into account the elements of heterogeneity, which allows predicting unplanned variants of behavior. The agents within the communities can have divergent interests, beliefs, intellectual and information resources, and other cognitive and social parameters. Mathematical modeling cannot provide a complete picture of the social system under inquiry, because use of differential equations tends to level heterogeneity, in this regard agent-based modeling gives an opportunity to describe nonlinear, complex, conflictogenic social processes in more detail.

Taking into account the fact that social and political sphere has a large proportion of irrational elements, we shall conclude that positivist methodology dominated by quantitative methods should be complemented with a set of qualitative methods which allow interpreting both rational and irrational actions. A wide range of important and interesting ideas is related to symbolic interactionism complemented with the methods of spectral analysis, which is based on determination of different carrying capacities of the system elements and which demonstrates heterogeneous character of the communicative field and, on this ground, shows different degree of inclusion into the communicative processes. Analysis of different types of social distance between individuals on the grounds of age, sex, education, ethnic and religious affiliation, etc. lets us find out the most effective influence mechanisms in terms of the special characteristics of overcoming the social distance in information field. The study of meanings and symbols, methods of their modification, frame analysis allow us discovering the structures that manage the human behavior.

Thus, for example, politics as a multifaceted phenomenon with a complex ontological structure should be analyzed non only basing on a conceptual perspective referring to the communication theories and cognitive psychology, but in the context of "integral equations", which let us work not with particular values, but with probabilistic system states, within which the parameters under inquiry will be more intensively manifested. Some important tendencies of information dissemination are predetermined by the realized limitations of the mass media invasion zone. Opinion clustering is more evident in zones with high communication density, where an elevated social tension is provided due to a smaller distance among the agents. Moreover, the level of mutual influence of the agents will be higher than the influence of the original information source, because the primary model experiences prominent transformations through complementing it with rumors and speculations. A working communicative practice is a logic chain of the following actions: drawing of attention, "reliability" creation, memory activation, emotional agitation, and message repetition. As it is well known, the chemical reaction catalyst provides the only possible and accelerated variant of interaction. If the signal repeats, 
the reaction goes faster and with less energy input. This natural pattern is reflected in the social communication processes, information technologies, and social and political interactions. Every message may be perceived as a vector: regarding both influence direction and dynamics of the information field.

\subsection{Complexity of Humanitarian Interactions: Methodology of the Media Discourse Analysis through Determination of the Density of an Information Stream}

In the context of analysis of the complexity of humanitarian interaction we shall take into account the fact that heterogeneous information streams and different modes of communication circulate in every certain social institution. Information streams are more saturated and intense within the relatively stable social institutions, than outside them. The density of an information stream increases with scientific and technical development, and a modern human being suffers from information overload, and experiences a significant information pressure, information "noise", and is caught in the "digital" and "virtual" crowd. A distinctive characteristic of informative and communicative processes in the contemporary society reflects the reaction of political systems to structural, organizational, resource, and other crises in the context of globalization of information society. I shall note that additional difficulties are created by interactions of different religious, ethnical, demographic and language communities in a conflict zone of opposition of political understandings, where mutual social confidence is needed in order to provide integrity of the social and humanitarian community. Moreover, "rapid societal transformations have a specific logic of influence from certain political, economic, and media structures, which influence one another in the context of conflict interaction, change priorities and create both destructive and constructive power mechanisms" 23 .

Methodology of conceptual language of policy analysis is based on the cognitive methods, suggested by the representatives of European and American schools of evolutionary concept-analysis: Q. Skinner, R. Koselleck, and others ${ }^{24}$. In particular, the idea of historical evolution of social concepts and the role of the turning periods in the birth of new conceptual complex is of great importance ${ }^{25}$.

The problem of social modeling, i.e. elaboration of specific variants of regional development is worth noticing.
As E. N. Ozhiganov argues, "in the Russian Federation there are specific characteristics, which should be taken into account when evaluating economic, demographic, and political environment of the regions. They are informal economy, undocumented income, and latent relations in the sphere of authority and administration" ${ }^{14}$.

Moreover, modeling is used as a prediction tool on the basis of a speculative experiment, which determines the mostadvantageous scenario in terms of goals achievement, and how this scenario should be implemented. It should be noted that there are not only speculative, but also rather real models. Thus, for example, some adequately selected focus groups outline a concept of political sentiments of the social groups which the researcher is interested in.

Content and degree of social security are directly related to the operation of all structures of the given society: economic, political, legal, and others, which is why the security itself has a complex structure. It includes social and economic, political, military, ecologic, food, information, psychological security. The following types of security are distinguished according to the space parameters and level of significance: global, international, national, and regional security. Modeling is used as a prediction tool on the basis of a speculative experiment, which determines the most advantageous scenario in terms of goals achievement, and how this scenario should be implemented.

Complex analysis of political, social and humanitarian mechanisms of the order formation includes the study of latent processes, which create additional threats to the system because of the activities of different actors with divergent goals.

Regionalization is intensified due to globalization and it is a permanent conflictogenic factor. The conflict configuration changed (they became more unique, less typical, local). All these factors should be interpreted, because there is no conflict management strategy at the regional level, the response is still situational, spontaneous, there is no system. A desire of regional ethnic elites to take power and, therefore, to use potential for disaffection can have serious consequences not only for the regional stability, but for the global society as well.

\section{Results}

\subsection{Theoretical Model as an Image of Preferable Future \\ Domestic political stability as a condition for regional and}


national security can be provided through maintaining the balance of interests of different social and ethnic groups. The researchers distinguish the following problems of the political process, which affect the state of the political system dynamic development: constitutional problems, economic problems, authority problems, culture problems ${ }^{26}$.

Analytical set of research mechanisms based on the principles of naturalistic philosophy can be a basis for system modeling of interaction among the main antagonists, taking into account their basic resources and behavior tactics, and can let us identify the most likely scenarios of development of a situation in a certain time period and on a certain territory. In this regard, the book "Atlas of Social and Political Problems, Threats, and Risks in the South of Russia" can be interesting as it comprehensively represents the situation in the Southern Federal District and in the cross-border regions ${ }^{15}$.

Elaboration of a social theory using science research methods aims at creation of a more complete professional knowledge for optimization of the state decisionmaking process. It is especially important to create such models which would more effectively reflect the special characteristics of policy and political behavior in a multiethnic macro-region. These special characteristics include probabilistic nature of political processes, formation of interest groups, recruitment of adherents, reconciliation of conflicting goals, and recognition of existence of different values. Possible conflicts in these conditions can have different nature: from destructive to those which spur the development. Naturally, modeling allows discovering possible tendencies which threaten the social system equilibrium, and creating certain countermeasure mechanisms against destructive influence of sufficiently influential political actors. But we need to take into account that creation of developmental models in order to ensure regional security should not rely on an entirely conflict-free society, because it is unreal and counterproductive. The most important in the modeling is to take into account the managerial resources of conflict interaction.

The problems of modeling in a scientific research of regional security are, first of all, related to the use of certain terminology. As T. Kuhn defines them, models are components of a so called disciplinary matrix. This category reflects two issues of the research activities. First and foremost, it is about patterns of certain solutions of the scientific problems, which are used as models, which in their turn, serve as a basis for working out of new tasks; secondly, it is a certain set of value orientations and a complex of recommendations to be implemented in practice $^{27}$.

Modeling in general and regional security modeling in particular have common theoretical roots with the general system theory, which is traditionally applied both in foreign and national Political Science. A series of the most advanced approaches to model the objects under inquiry was developed within this tradition ${ }^{28}$. The systematicity principle became a methodological program of the principles of regional modeling, despite significant differences in evaluations and certain methods, elaborated within this research area.

J. Galtung's integrative approach is worth mentioning. He suggests several stages of consistent interpretation of the relation between the empirical and the corresponding values. It lets us understand why empirical world is like it is, and foresee its possible future development. In this regard a model, an image of the desired future is created, and the potential possibility of its achievement is determined through elaboration and analysis of suggestions for changes to be made. Thus, it is about "creation of reality" or "correction of reality" 29 . In this case science and politics are united in their strive for achievement of a higher stage of correlation among what is observed, predicted and desired.

A scientific model includes a series of components, some of them are expressed in formalized language of scientific theories, others are included into it as implicit assumptions and conceptualizations, including those of axiological nature.

\subsection{Conceptual Tools of Scientific Methods in Social and Humanitarian Sciences}

Therefore, I shall identify the terms "model" and "theory" in the context of the topic of determination of possible use of scientific methods in social and humanitarian researches. These notions have the following definitions in the methodology of contemporary science. Model is a conceptual tool aimed at management of a modeled process or event, where the prediction, prognostic function serves an end of management. Theory, in comparison with model, is a more abstract and conceptual tool, aimed at explanation of the given processes and events ${ }^{30}$. Certain tools of interpretation of significant events precede the modeling of social and political processes in the spatial extent of certain regions. 
That is why case study is a necessary additional method of the study of social problems. In this regard, a model of social and political process is essentially a formalized expression of interpretation of political, economic, spiritual and ideological reality. The point of view of $P$. A. Sorokin is methodologically important: the model of a social phenomenon includes not only individuals or interactions among them. He assumes that characteristics of individuals and interactions are functionally connected, that is why they should not be separated one from another, because a model of processes of interaction among individuals is a social unit subject to the study ${ }^{31}$.

It is considered that models are objects, which are constructed in order to help understand real objects. They are often regarded as a simplified scheme of reality. Evidently, such approach to modeling is itself very simplified. Because when the Center for the Study of Security Problems, established at the Russian Academy of Sciences, puts forward a task of elaborating regional security models, it is supposed that these models should not only describe the situation in different sub-federal entities, but should they determine the target model characteristics, action cues, which would provide a sustainable balanced development of every region taking into account its special characteristics.

From methodological point of view, we shall distinguish static models, which are like a picture of a certain region at certain time, and dynamic models, which should represent "a picture of development of our constructs in time", taking into account the variations. One of the most complicated cognition problems is elaboration of terms for description of the semantic structure, which forms up as a conflict regional model. The model is considered as a conceptual tool, aimed at management of the modeled process, first of all ${ }^{32}$. Models limitations consist in the fact that they are static by definition, that is why and due to the processes of continuous changes of reality we need dynamic modeling of the interaction processes, which affect the security conditions and occurrence of new threats and risks. Social facts with external compulsory nature with respect to an individual, at the same time can be considered by an observer as socially constructed in the process of interactions among the actors, which have divergent intentions, beliefs, and goals. The principle of axiological neutrality is currently not much-in-demand, a scientist cannot be yet absolutely neutral, excluded from a certain social reality. The complexity theory is significantly important in natural sciences and, in particular, in Physics. The theory is about "the time arrow, the uncertainty", about the fact that "human social systems are the most complex of all the systems" ${ }^{\prime 3}$. Therefore, necessity for methodological pluralism is explained by the complexity theory, which is inclusively explained within the synergetic paradigm, which to a large extent is a link between studies of nature and studies of society as a synthesis of non-linear processes, moving from chaos to some sort of order and understood epistemologically on the basis of scientific analysis, including scientific and hermeneutic methods. Cognition of the complex should not be minimized to artificially formulated, "clean" methods of gaining insight into the knowledge.

Therefore, necessity to develop scientific research methods for social and humanitarian processes, and to elaborate different project social models is determined by objectively existing or potentially possible risks and threats, which can lead to a crisis situation in one or another region. Cognition in social and humanitarian sphere draws on multiparadigm principles in order to prevent or avoid such crisis situation. Nature and culture sciences cannot be in opposition, because they have the same scientific methods of cognition in common. Nevertheless, the process of cognition of social and humanitarian phenomena is a set of explanatory models for cognition of the complex, taking into account special characteristics of contemporary transformative social and humanitarian technologies and their engineering capabilities and basing on scientific methods and theories of exact sciences.

\section{References}

1. Outhwaite U. Laws and Explanations in Sociology. In: Deviatko I, editor. Models and Logics of a Sociological Research. Moscow; 1996 [in Russian].

2. Aidinian EM, Shipunova TV. Op. cit.; 37-38; 2000 [in Russian].

3. Bogdanov AA. Tectology. Universal organizational science. Moscow; 1989 [in Russian].

4. Atamanchuk GV. Public Administration Theory. Moscow; 1998 [in Russian].

5. Romanov VL. Social Self-Organization and National Identity. Moscow; 2000 [in Russian].

6. Shabrov OF. Political Management. Problems of Stability and Development. Moscow; 1997 [in Russian].

7. Ovcharenko AN; 2007.

8. Kultygin VP. Problems of Social Cognition at the Turn of Millennium. Problems of Theoretical Sociology. Saint Pe- 
tersburg: Chemical Research Institute of the Saint Petersburg State University. 2003; 4:11-23 [in Russian].

9. Vasilkova VV. Order and Chaos in Social System Development. Saint Petersburg: Lan; 1999 [in Russian].

10. Prigozhin I, Nikolisa G. Cognition of complex; 2002.

11. Berger P, Luckmann T. The Social Construction of Reality: a Treatise in the Sociology of Knowledge. Transl from English by Rutkevich E. Moscow: Academiya-Centr; Medium. 1995.

12. Popper K, 2000.

13. Ritzer G, Sovremennyesotsiologicheskieteorii [Contemporary Sociological Theory] Transl. from English by Boikov A, Lisitsina A. St.Petersburg: Peter; 2002. [in Russian]

14. Ozhiganov EN. Strategic Policy Analysis: Theoretical Fundamentals and Methods. Moscow. 2006; 168 [in Russian].

15. Matishov GG. Atlas of Social and Political Problems, Threats, and Risks in the South of Russia. Rostov-on-Don: Southern Scientific Center of the Russian Academy of Sciences Press; 2006-2013 [in Russian].

16. Neklessa AI. Cognition and Construction of the World (Values, Knowledge, Action: Genesis and Dynamics of the Forms). Materials of the Scientific Seminar. Moscow, 2007; 1:6 [in Russian].

17. Boronoev AO. Problems of Theoretical Sociology. Vol. 4 : interacademic collection. Saint Petersburg: Chemical Research Institute of Saint Petersburg State University. 2003; 16 [in Russian].

18. Latane B, Wolf S. The Social Impact of Majorities. Psychological Review. 1981; 13, 343, 438.

19. Yakunin V, Sylakshin S. About the Significance of Factor Analysis at the Time of Formation of State Policy. Power. 2006. p. 64-9 [in Russian].

20. Libicki MC. Cyber Deterrence and Cyber War. RAND Corporation; 2009.
21. Ovchinsky V, Larina E. Cold War 2.0. In Cold War 2.0. Strategy of the Russian Victory. Moscow: Izborsky Club. Book World. 2015; 49-104 [in Russian].

22. Nowak A, Szamrej J, Latané B. From Private Attitude to Public Opinion: a Dynamic Theory of Social Impact. Psychological Review. 1990; 97:362-76.

23. Yurchenko NN. Concepts of Modernization in a Conflict Field of Political Interpretations. State Service. 2011; 5:8790 [in Russian].

24. Zavershinsky KF. Methodological Complementarity in a Research of Symbolic Matrix of the Dynamic of Political Institutes. Polis. 2003; 1 [in Russian].

25. Koselleck R. Futures Past: on the Semantic of Historical Time. Cambridge and London; 1985.

26. Perepelkin L. State Ethnic Policy and Security Problems in the Ethnic Sphere. Conflict - Dialog - Collaboration. 2000; 2:18 [in Russian].

27. Kuhn T. Structure of Scientific Revolutions. Moscow. 1983; 231, 234 [in Russian].

28. BaskakovaYu M, Andronova IV. Influence of the Access to Information on the Models of Political Participations in Russian Regions. Information and Communication Technologies. 2006; 4 [in Russian].

29. Galtung J. 1999.

30. PlotinskyYu M. Models of Social Processes. Moscow. 2001; 87 [in Russian].

31. Sorokin P. A System of Sociology. Vol. 1. Social Analytics: Theory of the Structure of the Simplest Social Phenomenon. Moscow. 1993; 140 [in Russian].

32. Yurchenko IV. Regional Security as the Subject of Analysis within Conflict Resolution Studies (Apology of Methodological Pluralism). Polis.2007; 6:129 [in Russian].

33. Kultigin VP; 2000. 